\title{
Inaugural Editorial for Otorhinolaryngology-Head and Neck Surgery
}

\author{
Chin-Lung Kuo* \\ Department of Otolaryngology, Taoyuan Armed Forces General Hospital; Institute of Brain Science, National Yang-Ming University, Taiwan
}

It is my great honor to assume the role of founding Editor-in-Chief for Otorhinolaryngology-Head and Neck Surgery (OHNS). In accepting this role, my goal is to help OHNS establish itself as a premier academic journal. OHNS has a mission to promote scientific research for the benefit of humanity and to advance the practice of otorhinolaryngology and of head and neck surgery. Our vision is to become a reliable journal that provides high-quality scientific information and that is a leader in terms of attracting the best research and having the greatest impact.

OHNS provides a new avenue for investigators, clinicians, and trainees to publish their research. OHNS is a bimonthly journal with a vigorous, timely peer-review policy and a rapid publication process. OHNS is also part of the OA Text Network, a family of open-access journals that includes 45 specialty journals. Individually and collectively, we believe that open access is well aligned with the goals of science, and we further believe that increased availability of research data can benefit both medical research and science education. OHNS aims to bring about a change in the way that modern scholarly communication is conducted in order to accelerate scientific discoveries and to help disseminate research to a wider readership, gain media attention, and demonstrate professional achievement through publication.

The scope of OHNS is broad, encompassing all otorhinolaryngologyhead and neck surgery subspecialties. OHNS will feature original research (negative and positive results), analyses, reviews (systematic, narrative, and mini-reviews), letters to the editor, case reports, news, practice updates, thought-provoking editorials, expert opinion articles, forward-looking perspectives, commentaries, images, and book reviews. OHNS will also publish papers that cover in vitro and animal studies. Subject areas include, but are not limited to, otology, rhinology, laryngology, head and neck surgery, chronic ear infection, hearing loss, cochlear implants, sinonasal disorders, rhinitis and rhinosinusitis, phonosurgery, thyroid surgery, head and neck cancer, cranio-maxillofacial surgery, facial plastic surgery, snoring and sleep medicine, salivary

Copyright: (C2016 Kuo CL. This is an open-access article distributed under the terms of the Creative Commons Attribution License, which permits unrestricted use, distribution, and reproduction in any medium, provided the original author and source are credited. gland disorders, otoneurology, audiology, and pediatric otolaryngology. The journal will also accept contributions that present innovative and improved methods or resources that advance basic research. OHNS will keep readers informed about new and emerging trends quickly by periodically publishing special issues. Authors are encouraged to submit rigorous and methodologically sound systematic reviews and meta-analyses.

It is my great honor to work with the OHNS editorial board members and senior editors. All editorial board members are all internationally recognized researchers who have committed to devoting their time and effort to developing and promoting the journal. I will rely heavily on them to review manuscripts and make sound recommendations regarding suitability for publication. Editorial board members will also be able to propose and organize timely special issues in their subfields. As research and science continues to evolve, I plan to recruit additional editorial board members in order to enhance the expertise of the journal's senior staff. As editor-in-chief, I will closely work with editors, editorial board members, reviewers, and authors, and I am committed to placing a high priority on the publication of high-quality research.

I wish to close this inaugural editorial by inviting authors to submit their exciting research discoveries to OHNS, and by thanking editors, reviewers, authors, and readers for their support. Any papers that are submitted, either individually or collaboratively, are greatly appreciated and will make a substantial contribution to the development and success of OHNS. With your assistance, we will build a journal of excellence to serve the research community of otorhinolaryngology-head and neck surgery with the ultimate goal of improving lives.

Chin-Lung Kuo, MD

Editor-in-Chief

Otorhinolaryngology-Head and Neck Surgery
Correspondence to: Chin-Lung Kuo, Department of Otolaryngology, Taoyuan Armed Forces General Hospital; Institute of Brain Science, National Yang-Ming University, Taiwan, ROC, Tel: 886-9-19681078; E-mail: drkuochinlung@gmail.com Received: April 01, 2016; Accepted: April 02, 2016; Published: April 04, 2016 